\title{
AVERAGES OF COEFFICIENTS OF A CLASS OF DEGREE 3 $L$-FUNCTIONS
}

\author{
BINGRONG HUANG, YONGXIAO LIN, AND ZHIWEI WANG
}

\begin{abstract}
In this note, we give a detailed proof of an asymptotic for averages of coefficients of a class of degree three $L$-functions which can be factorized as a product of a degree one and a degree two $L$-functions. We emphasize that we can break the $1 / 2$-barrier in the error term, and we get an explicit exponent.
\end{abstract}

\section{INTRODUCTION}

In number theory, one of the most important and central problem is to give an asymptotic formula as accurate as possible for the sum

$$
A(X)=\sum_{n \leqslant X} a(n)
$$

where $a(n)$ is an arithmetic function. In many cases, this problem can be transformed to the study of the following generating series

$$
L(s)=\sum_{n=1}^{\infty} \frac{a(n)}{n^{s}}
$$

which satisfies valuable analytic properties. Here we just list two classical examples.

- $a(n)=\Lambda(n)$ is the von Mangoldt function, then this is the well-known prime number theorem.

- $a(n)=\tau_{k}(n), k \geqslant 2$ is the divisor function, then this is the divisor problem. One may see for example the work of Atkinson [1] for the case $k=3$.

In this paper, we are interested in investigating the case where $a(n)=\lambda_{F}(n)$, the coefficients of a degree $d$ automorphic $L$-function (see Section 2.1). We set

$$
A(X, F)=\sum_{n \leqslant X} \lambda_{F}(n), \quad L(s, F)=\sum_{n \geqslant 1} \frac{\lambda_{F}(n)}{n^{s}} .
$$

For $A(X, F)$, one may expect that

$$
A(X, F)=\operatorname{Res}_{s=1} \frac{L(s, F)}{s} X+O\left(X^{\theta}\right)
$$

Date: March 10, 2020.

2010 Mathematics Subject Classification. 11N37, 11F30.

Key words and phrases. Fourier coefficient, $L$-function, short interval, exponent pair.

B. H. was supported by the Young Taishan Scholars Program of Shandong Province (Grant No. tsqn201909046) and Qilu Young Scholar Program of Shandong University. Y. L. was partially supported by a DFG-SNF lead agency program grant (Grant 200020L_175755). Z. W. was supported by China Postdoctoral Science Foundation (No. 2019M652354) and National Natural Science Foundation of China (No. 11901348). 
with $\theta$ as small as possible. In particular, this would imply

$$
\sum_{X<n \leqslant X+Y} \lambda_{F}(n)=\operatorname{Res}_{s=1} \frac{L(s, F)}{s} Y+o(Y)
$$

for $Y>X^{\theta+\varepsilon}$, for any fixed $\varepsilon>0$.

Under the generalized Riemann hypothesis (GRH), we may take $\theta=1 / 2$. To surpass the GRH with some $\theta<1 / 2$ is an interesting and challenging problem. For general series of degree 2 , this is already achieved with $\theta=1 / 3$. Friedlander and Iwaniec [5] proved a quite general result that for $d \geqslant 2$, (1.1) holds for $\theta=\frac{d-1}{d+1}\left(\geqslant \frac{1}{2}\right.$ for $\left.d \geqslant 3\right)$ under some local assumptions.

For the case of degree " $1+1+1$ " when $L(s, F)$ factors completely into degree $1 L$-functions, it was showed that we can take $\theta=\frac{37}{75}<\frac{1}{2}$ in (1.1) (see e.g. Friedlander and Iwaniec [5]). They also announced that the other case of degree " $1+2$ " can also be successfully treated with additional arguments. For $d=6$, recently together with Sun, the second named author [11] was able to beat the bound $\frac{6-1}{6+1}$ (under Ramanujan-Selberg) for a class of $\mathrm{GL}_{3} \times \mathrm{GL}_{2}$ automorphic forms, namely, for $F=f \otimes \phi$, Rankin-Selberg convolution of a $\mathrm{GL}_{3}$ cusp form $f$ and a $\mathrm{GL}_{2}$ cusp form $\phi$. Subsequently, the first named author [7] was able to make further progress on another important case, by improving the error term $O\left(X^{3 / 5}\right)$ for $F=\phi \times \phi$ for the case $d=4$. Specially, he remarked that the method in [7] is applicable to the degree " $1+2$ " case. Here, crucial ingredients in these works are factorazation of the coefficients: in Friedlander-Iwaniec's case $\lambda_{F}(n)=\sum_{n_{1} n_{2} n_{3}=n} \chi_{1}\left(n_{1}\right) \chi_{2}\left(n_{2}\right) \chi_{3}\left(n_{3}\right)$; in Lin-Sun's case $\lambda_{F}(n)=\sum_{m_{1}^{2} m_{2}=n} \lambda_{f}\left(m_{1}, m_{2}\right) \lambda_{\phi}\left(m_{2}\right)$, while in Huang's case $\lambda_{F}(n)=$ $\sum_{\ell m=n} \lambda_{\text {sym }^{2}}(m)$.

In this paper, we shall consider $A(X, F)$ for another case of degree 3 , when $F=1 \boxplus \phi$; that is

$$
L(s, F)=L(s, 1 \boxplus \phi)=\zeta(s) L(s, \phi) .
$$

Here $\phi$ is a holomorphic cusp form. We give a first detailed proof of (1.1) with $\theta<\frac{1}{2}$, by combining the methods of Friedlander-Iwaniec in [5] and the first named author in [7].

Theorem 1. With the notation as above. The asymptotic formula

$$
A(X, 1 \boxplus \phi)=L(1, \phi) X+O\left(X^{1 / 2-\delta+\varepsilon}\right)
$$

holds for $\delta=4 / 739 \approx 0.00541 \ldots$ and any fixed $\varepsilon>0$.

An immediate consequence of this theorem is the following asymptotic formula for averages of those coefficients in rather short intervals.

Corollary 1. With the notation as above. We have

$$
\sum_{X<n \leqslant X+Y} \lambda_{1 \boxplus \phi}(n)=L(1, \phi) Y+O\left(Y^{1-\varepsilon}\right)
$$

as long as $Y \gg X^{1 / 2-\delta}$, for any $\delta<4 / 739 \approx 0.00541 \ldots$.

The functions $n \mapsto \tau_{3}(n)$ and $n \mapsto \lambda_{1 \boxplus \phi}(n)$ are the Hecke eigenvalues of certain noncuspidal automorphic representation of $\mathrm{GL}_{3, \mathbb{Q}}$, that is, the isobaric representation $1 \boxplus 1 \boxplus 1$ and $1 \boxplus \pi_{\phi}$. The methods of $[1,5,7]$ and of the present paper can be generalized straightforwardly to prove that the average of the $n$-th Hecke eigenvalue function of any fixed non-cuspidal 
automorphic representation of $\mathrm{GL}_{3, \mathbb{Q}}$ has exponent $<1 / 2$ in the error term. Extending this further to cuspidal $\mathrm{GL}_{3, \mathbb{Q}}$-representations is an interesting and challenging question.

Remark 1. Under the generalized Ramanujan conjecture, it is clear that the same result can be proved for $\phi$ being a Hecke-Maass cusp form by our method.

Remark 2. (1) There is an arithmetic analogue of the questions (1.1) and (1.2). Indeed, one can study the distribution of $\lambda_{F}(n)$ by also asking how uniform the coefficients $\lambda_{F}(n)$ are distributed, when we vary $n$ among arithmetic progressions $a m+q$. Namely, do we have

$$
\sum_{\substack{n \leqslant X \\ n \equiv a \bmod q}} \lambda_{F}(n)-\frac{1}{\varphi(q)} \sum_{\substack{n \leqslant X \\(n, q)=1}} \lambda_{F}(n) \ll_{A} \frac{X}{q}(\log X)^{-A}
$$

for $q \leqslant X^{\vartheta}$, with $\vartheta$ as large as possible? Here $\varphi$ is the Euler function and the exponent $\vartheta$ is called the level of distribution. It is predicted that one can take $\vartheta=1-\varepsilon$. In general, for $F$ an $\mathrm{GL}_{\mathrm{d}}$ automorphic form, this can be studied by detecting the condition $n \equiv a \bmod q$ using additive characters and then applying the Voronoï summation, which would transform the sum in question to a dual sum of length roughly $q^{d} / X$ and to the dual Hecke eigenvalue $\overline{\lambda_{F}(n)}$, but twisted by a $(d-1)$-dimensional hyper-Kloosterman sum $\mathrm{Kl}_{d}($ an; $q)$, upon which an application of Deligne's estimate will produce a level of distribution $\frac{2}{d+1}$ for the sum above. Such an exponent is regarded as the trivial level of distribution for $\lambda_{F}(n)$. To beat this exponent for various $\lambda_{F}$ is an active area of research. For example, results breaking the 1/2-barrier for the level $\vartheta$ which is also the same barrier of the well-known BombieriVinogradov theorem, are known for several special cases:

- when $\lambda_{F}(n)=\tau(n)$, we can take $\vartheta=2 / 3-\varepsilon$, a classical result of Selberg which remains the best to date;

- when $\lambda_{F}(n)=\tau_{3}(n)$, one can take $\vartheta=1 / 2+1 / 230-\varepsilon$, corresponding to the groundbreaking result of Friedlander-Iwaniec [4].

- when $\lambda_{F}(n)=\lambda_{1 \boxplus \phi}(n)$, one has $\vartheta=1 / 2+1 / 102-\varepsilon$, which was proved by KowalskiMichel-Sawin [10], building on their breakthrough on estimates for bilinear forms in hyper-Kloosterman sums.

(2) Theorem 1 (and its corollary) is exactly an Archimedian analogue of (1.3) when $F=$ $1 \boxplus \phi$, proved by Kowalski-Michel-Sawin.

Throughout the paper, $\varepsilon$ is an arbitrarily small positive number; all of them may be different at each occurrence.

\section{PRELIMINARIES}

2.1. $L$-functions. In general, the $L$-function $L(s, F)$ satisfies the following conditions:

(1). We have the Euler product of degree $d$

$$
L(s, F)=\sum_{n \geqslant 1} \frac{\lambda_{F}(n)}{n^{s}}=\prod_{p} \prod_{j=1}^{d}\left(1-\frac{\alpha_{j}(p)}{p^{s}}\right)^{-1}
$$

with $\lambda_{f}(1)=1$. The series and Euler products are absolutely convergent for $\operatorname{Re} s>1$. The sequence $\left\{\lambda_{F}(n)\right\}_{n \geqslant 1}$ are called coefficients of $L(s, F)$, and the $\alpha_{j}(p), 1 \leqslant j \leqslant d$ satisfying $\left|\alpha_{j}(p)\right|<p$ for all $p$, are called the local parameters of $L(s, F)$ at $p$. 
(2). We have the gamma factor defined by

$$
\gamma(s, F)=\pi^{-d s / 2} \prod_{j=1}^{d} \Gamma\left(\frac{s+\kappa_{j}}{2}\right)
$$

where the numbers $\kappa_{j} \in \mathbb{C}$ satisfying $\operatorname{Re} \kappa_{j}>-1$, are called the local parameters of $L(s, F)$ at infinity.

(3). There exists an integer $q(F) \geqslant 1$, called the conductor of $L(s, F)$, satisfying $\alpha_{j}(p) \neq 0$ for $1 \leqslant j \leqslant d, p \nmid q(F)$ such that we have the functional equation

$$
\Lambda(s, F)=\varepsilon(F) \Lambda(1-s, \bar{F})
$$

where $\bar{F}$ is the dual form of $f$ for which $\lambda_{\bar{F}}(n)=\overline{\lambda_{F}(n)}, \gamma(s, \bar{F})=\gamma(s, F), q(\bar{F})=q(F)$, and $\varepsilon(F)$ is the root number of $L(s, F)$ satisfying $|\varepsilon(F)|=1$. Here $\Lambda(s, F)$ is called the complete $L$-function defined by

$$
\Lambda(s, F)=q(F)^{s / 2} \gamma(s, F) L(s, F) .
$$

For more details, we refer the reader to the book of Iwaniec-Kowalski [8, Chapter 5.1].

\subsection{Functional equation.}

Lemma 2.1. For $\operatorname{Re} s>1$, we have

$$
L(1-s, 1 \boxplus \phi)=w \gamma(s) L(s, 1 \boxplus \phi)
$$

where $w=i^{k}$ and $\gamma(s)$ satisfies

$$
\gamma(\sigma-i t)=\bar{\omega}(Q t)^{3(\sigma-1 / 2)}\left(\frac{\mathrm{e}}{Q t}\right)^{3 i t}\left\{1+O\left(\frac{1}{t}\right)\right\}
$$

for $\sigma>1 / 2, t>1$, and

$$
Q=\frac{1}{2 \pi}, \quad \omega=\mathrm{e}\left(\frac{2 k-3}{8}\right), \text { where } \mathrm{e}(u)=\mathrm{e}^{2 \pi i u} .
$$

Proof. First by the functional equation

$$
\Lambda(s, 1 \boxplus \phi)=\pi^{-\frac{s}{2}} \Gamma\left(\frac{s}{2}\right) \zeta(s) \cdot(2 \pi)^{-s} \Gamma\left(s+\frac{k-1}{2}\right) L(s, \phi)=i^{k} \Lambda(1-s, 1 \boxplus \phi),
$$

we can write the functional equation of the Dirichlet generating series for the coefficient $\lambda_{1 \boxplus \phi}(n)$ as follows:

$$
\begin{aligned}
L(1-s, 1 \boxplus \phi) & =i^{k} \frac{\pi^{-\frac{s}{2}} \Gamma\left(\frac{s}{2}\right)(2 \pi)^{-s} \Gamma\left(s+\frac{k-1}{2}\right)}{\pi^{-\frac{1-s}{2}} \Gamma\left(\frac{1-s}{2}\right)(2 \pi)^{-1+s} \Gamma\left(1-s+\frac{k-1}{2}\right)} L(s, 1 \boxplus \phi) \\
& =w \gamma(s) L(s, 1 \boxplus \phi)
\end{aligned}
$$

where

$$
w=i^{k}, \quad \gamma(s)=\pi^{\frac{1}{2}-s}(2 \pi)^{1-2 s} \frac{\Gamma\left(\frac{s}{2}\right)}{\Gamma\left(\frac{1-s}{2}\right)} \cdot \frac{\Gamma\left(s+\frac{k-1}{2}\right)}{\Gamma\left(1-s+\frac{k-1}{2}\right)}
$$


Next, we shall write $\gamma(s)$ in the form

$$
\gamma(s)=\left(\pi^{-m} D\right)^{s-\frac{1}{2}} \prod_{j=1}^{m} \Gamma\left(\frac{s+\kappa_{j}}{2}\right) \Gamma\left(\frac{1-s+\kappa_{j}}{2}\right)^{-1}
$$

where $D$ is the conductor, and the "spectral parameters" $\kappa_{j}$ are complex numbers having $\operatorname{Re} \kappa_{j}>0$. And $k=\sum_{j=1}^{m} \kappa_{j}$ is the weight.

Note that $\Gamma(z) \Gamma\left(z+\frac{1}{2}\right)=2^{1-2 z} \pi^{\frac{1}{2}} \Gamma(2 z)$. Taking $z=\frac{s+\frac{k-1}{2}}{2}$, we obtain

$$
\Gamma\left(s+\frac{k-1}{2}\right)=2^{s+\frac{k-3}{2}} \pi^{-\frac{1}{2}} \Gamma\left(\frac{s+\frac{k-1}{2}}{2}\right) \Gamma\left(\frac{s+\frac{k+1}{2}}{2}\right) .
$$

With the notation of $\gamma(s)$ in (2.2), we derive

$$
\begin{aligned}
\gamma(s) & =\pi^{\frac{1}{2}-s}(2 \pi)^{1-2 s} \frac{\Gamma\left(\frac{s}{2}\right)}{\Gamma\left(\frac{1-s}{2}\right)} \cdot \frac{2^{s+\frac{k-3}{2}} \pi^{-\frac{1}{2}} \Gamma\left(\frac{s+\frac{k-1}{2}}{2}\right) \Gamma\left(\frac{s+\frac{k+1}{2}}{2}\right)}{2^{1-s+\frac{k-3}{2}} \pi^{-\frac{1}{2}} \Gamma\left(\frac{1-s+\frac{k-1}{2}}{2}\right) \Gamma\left(\frac{1-s+\frac{k+1}{2}}{2}\right)} \\
& =\left(\pi^{-3}\right)^{s-\frac{1}{2}} \prod_{j=1}^{3} \Gamma\left(\frac{s+\kappa_{j}}{2}\right) \Gamma\left(\frac{1-s+\kappa_{j}}{2}\right)^{-1},
\end{aligned}
$$

which is of the form (2.3) with $m=3, D=1$ and $\kappa_{1}=0, \kappa_{2}=\frac{k-1}{2}, \kappa_{2}=\frac{k+1}{2}$. Hence, by the argument of Friedlander-Iwaniec [5, Section 1], we complete the proof of Lemma 2.1 with $Q=(2 \pi)^{-1}, \omega=\mathrm{e}\left(\frac{2 k-3}{8}\right)$.

On taking $s=1+\varepsilon-i t$, Lemma 2.1 yields

$$
L(-\varepsilon+i t, 1 \boxplus \phi)=w \bar{\omega}\left(\frac{t}{2 \pi}\right)^{3\left(\frac{1}{2}+\varepsilon\right)}\left(\frac{t}{2 \pi \mathrm{e}}\right)^{-3 i t} L(1+\varepsilon-i t, 1 \boxplus \phi)\left\{1+O\left(\frac{1}{t}\right)\right\} .
$$

\section{Proof of Theorem 1}

In this section, we consider the sum $A(X, 1 \boxplus \phi)=\sum_{n \leqslant X} \lambda_{1 \boxplus \phi}(n)$, that is we have

$$
L(s, 1 \boxplus \phi)=\zeta(s) L(s, \phi)=\zeta(s) \sum_{n=1}^{\infty} \frac{\lambda_{\phi}(n)}{n^{s}}
$$

for $\operatorname{Re}(s)>1$, where $\phi$ is a $\mathrm{GL}_{2}$ automorphic form and $\lambda_{\phi}(n)$ is defined by

$$
\phi(z)=\sum_{n \geqslant 1} \lambda_{\phi}(n) n^{\frac{k-1}{2}} \mathrm{e}(n z), \quad \mathrm{e}(z)=\mathrm{e}^{2 \pi i z} .
$$

Assume $\phi$ is holomorphic, i.e., $\phi \in H_{k}\left(\mathrm{SL}_{2}(\mathbb{Z})\right)$, an orthogonal basis of the space of holomorphic cusp forms of weight $k$ and level 1 , with $k \geqslant 12$ even. We first approximate $A(X, 1 \boxplus \phi)$ by a smooth sum. Let

$$
Y=X^{1 / 2-\delta}, \quad \text { for some } \delta \in(0,1 / 10)
$$

Let $W$ be a smooth function with support $\operatorname{supp} W \in[1 / 2-Y / X, 1+Y / X]$ such that $W(u)=$ $1, u \in[1 / 2,1]$ and $W(u) \in[0,1], u \in[1 / 2-Y / X, 1 / 2] \cup[1,1+Y / X]$, and $W^{(k)}(u) \ll(X / Y)^{k}$. 
Therefore we have the approximating forumla

$$
\begin{aligned}
\sum_{X / 2<n \leqslant X} \lambda_{1 \boxplus \phi}(n)= & \sum_{X / 2-Y<n<X+Y} \lambda_{1 \boxplus \phi}(n) W\left(\frac{n}{X}\right) \\
& +O\left(\sum_{X / 2-Y<n<X / 2}\left|\lambda_{1 \boxplus \phi}(n)\right|+\sum_{X<n<X+Y}\left|\lambda_{1 \boxplus \phi}(n)\right|\right) \\
= & \sum_{n \geqslant 1} \lambda_{1 \boxplus \phi}(n) W\left(\frac{n}{X}\right)+O\left(X^{1 / 2-\delta+\varepsilon}\right)
\end{aligned}
$$

where we have used Deligne's bound $\lambda_{1 \boxplus \phi}(n) \ll \sum_{\ell m=n} m^{\varepsilon} \ll n^{\varepsilon}$ when $\phi$ is holomorphic for the error terms.

Next we only need to show

$$
\sum_{n \geqslant 1} \lambda_{1 \boxplus \phi}(n) W\left(\frac{n}{X}\right)=L(1, \phi) \tilde{W}(1) X+O\left(X^{1 / 2-\delta+\varepsilon}\right)
$$

where $\tilde{W}(s)=\int_{0}^{\infty} W(x) x^{s-1} \mathrm{~d} x$ is the Mellin transform of $W$. Since by inserting (3.2) into (3.1) and then by iteration, we get

$$
\sum_{n \leqslant X} \lambda_{1 \boxplus \phi}(n)=2 L(1, \phi) \tilde{W}(1) X+O\left(X^{1 / 2-\delta+\varepsilon}\right) .
$$

Then Theorem 1 follows immediately from the estimate $\tilde{W}(1)=1 / 2+O(Y / X)$.

Now we estimate the sum $\sum_{n \geqslant 1} \lambda_{1 \boxplus \phi}(n) W\left(\frac{n}{X}\right)$ in (3.2). By the inverse Mellin transform

$$
W(u)=\frac{1}{2 \pi i} \int_{(2)} \tilde{W}(s) u^{-s} \mathrm{~d} s
$$

we get

$$
\sum_{n \geqslant 1} \lambda_{1 \boxplus \phi}(n) W\left(\frac{n}{X}\right)=\frac{1}{2 \pi i} \int_{(2)} \tilde{W}(s) L(s, 1 \boxplus \phi) X^{s} \mathrm{~d} s .
$$

We then move the integration to the parallel segment with $\operatorname{Re} s=\sigma=-\varepsilon$. We pass pole at $s=1$ with residue $\operatorname{Res}_{s=1} L(s, 1 \boxplus \phi)=L(1, \phi)$ since $L(s, 1 \boxplus \phi)=\zeta(s) L(s, \phi)$. Hence we obtain

$$
\sum_{n \geqslant 1} \lambda_{1 \boxplus \phi}(n) W\left(\frac{n}{X}\right)=L(1, \phi) \tilde{W}(1) X+\frac{1}{2 \pi i} \int_{(-\varepsilon)} \tilde{W}(s) L(s, 1 \boxplus \phi) X^{s} \mathrm{~d} s .
$$

We denote by $I(X)$ the second term with integration on the right hand side of (3.3). Inserting a dyadic smooth partition of unit to the $t$-integral, we get

$$
I(X)=\sum_{T \text { dyadic }} I(X, T)
$$

where

$$
I(X, T):=\frac{X^{-\varepsilon}}{2 \pi} \int_{\mathbb{R}} X^{i t} \tilde{W}(-\varepsilon+i t) L(-\varepsilon+i t, 1 \boxplus \phi) V\left(\frac{t}{T}\right) \mathrm{d} t
$$


for some fixed $V$ with compact support. For $\tilde{W}(s)$, by integration by parts, we have the estimate for any $k \geqslant 1$

$$
\tilde{W}(s)=\frac{(-1)^{k}}{s(s+1) \cdots(s+k-1)} \int_{0}^{\infty} W^{(k)}(u) u^{s+k-1} \mathrm{~d} u \ll \frac{1}{|s|^{k}}\left(\frac{X}{Y}\right)^{k-1},
$$

since $\operatorname{supp} W^{(k)} \in[1 / 2-Y / X, 1 / 2] \cup[1,1+Y / X]$. This estimate allows us to truncate the $t$-integral of $I(X, T)$ at $T \ll X^{1+\varepsilon} / Y$. In addition, by the upper bounds $L(-\varepsilon+i t, 1 \boxplus \phi) \ll$ $(1+|t|)^{3 / 2+\varepsilon}$ and $(3.5)$ with $k=1$, we deduce that

$$
I(X, Y) \ll X^{\varepsilon} T^{3 / 2+\varepsilon} \ll Y
$$

if $T \ll Y^{2 / 3-\varepsilon}$. Therefore, by the above arguments, we may impose a constraint $Y^{2 / 3-\varepsilon} \ll$ $T \ll X^{1+\varepsilon} / Y$ in (3.4) with an admissible error term. We only consider positive $T$ 's, since negative $T$ 's can be handled similarly. Next, for $I(X, T)$, by the first equality in (3.5) with $k=1$, we get

$$
\begin{aligned}
I(X, T) & =-\frac{X^{-\varepsilon}}{2 \pi} \int_{1 / 3}^{3} W^{\prime}(u) u^{-\varepsilon} \int_{\mathbb{R}} \frac{(X u)^{i t}}{-\varepsilon+i t} L(-\varepsilon+i t, 1 \boxplus \phi) V\left(\frac{t}{T}\right) \mathrm{d} t \mathrm{~d} u \\
& \ll \frac{X^{-\varepsilon}}{T} \sup _{u \in[1 / 3,3]}\left|\int_{\mathbb{R}}(X u)^{i t} L(-\varepsilon+i t, 1 \boxplus \phi) V\left(\frac{t}{T}\right) \mathrm{d} t\right| .
\end{aligned}
$$

Hence, in the following, we only need to consider $J(X, T)$ which is defined by

$$
J(X, T):=\int_{\mathbb{R}} X^{i t} L(-\varepsilon+i t, 1 \boxplus \phi) V\left(\frac{t}{T}\right) \mathrm{d} t .
$$

To estimate $J(X, T)$, we shall apply functional equation for $L(-\varepsilon+i t, 1 \boxplus \phi)$ to change the variable $s=-\varepsilon+i t$ into $1-s=1+\varepsilon-i t$.

By inserting the functional equation (2.5) into (3.7), it follows that

$$
\begin{aligned}
J(X, T)= & \int_{\mathbb{R}} X^{i t} w \bar{\omega} \cdot\left(\frac{t}{2 \pi}\right)^{3\left(\frac{1}{2}+\varepsilon\right)}\left(\frac{t}{2 \pi \mathrm{e}}\right)^{-3 i t} L(1+\varepsilon-i t, 1 \boxplus \phi) V\left(\frac{t}{T}\right) \mathrm{d} t \\
& +O\left(\frac{1}{T} \cdot T^{3 / 2+\varepsilon} \cdot T\right) \\
\ll & T^{3 / 2+\varepsilon}\left|\int_{\mathbb{R}} \sum_{n \geqslant 1} \frac{\lambda_{1 \boxplus \phi}(n)}{n^{1+\varepsilon-i t}} X^{i t}\left(\frac{t}{2 \pi \mathrm{e}}\right)^{-3 i t} V_{1}\left(\frac{t}{T}\right) \mathrm{d} t\right|+T^{3 / 2+\varepsilon},
\end{aligned}
$$

for some smooth compactly supported function $V_{1}$.

Changing the order of the integral of summation above, and making a change of variable $t=T \xi$, we get

$$
J(X, T) \ll T^{5 / 2+\varepsilon}\left|\sum_{n \geqslant 1} \frac{\lambda_{1 \boxplus \phi}(n)}{n^{1+\varepsilon}} \int_{\mathbb{R}} V_{1}(\xi) \mathrm{e}^{i T \xi \log \left(n X\left(\frac{T \xi}{2 \pi \mathrm{e}}\right)^{-3}\right)} \mathrm{d} \xi\right|+T^{3 / 2+\varepsilon} .
$$

For the above integral over $\xi$, by the stationary phase method with

$$
h(\xi)=T \xi \log \left(\frac{n X}{\left(\frac{T \xi}{2 \pi \mathrm{e}}\right)^{3}}\right), \quad \xi_{0}=\frac{2 \pi(n X)^{1 / 3}}{T}, \quad h\left(\xi_{0}\right)=3 T \xi_{0}, \quad h^{\prime \prime}\left(\xi_{0}\right)=-\frac{3 T}{\xi_{0}} \asymp T,
$$


we get (see e.g. [2, Proposition 8.2])

$$
\begin{aligned}
\int_{\mathbb{R}} V_{1}(\xi) \mathrm{e}^{i T \xi \log \left(n X\left(\frac{T \xi}{2 \pi \mathrm{e}}\right)^{-3}\right)} \mathrm{d} \xi & =\frac{\mathrm{e}^{i h\left(\xi_{0}\right)}}{T^{1 / 2}} W_{1}\left(\xi_{0}\right)+O\left(\frac{1}{T^{2020}}\right) \\
& =\frac{\mathrm{e}\left(3(n X)^{1 / 3}\right)}{T^{1 / 2}} W_{2}\left(\frac{n}{T^{3} / X}\right)+O\left(\frac{1}{T^{2020}}\right),
\end{aligned}
$$

for some inert functions $W_{1}, W_{2}$, and hence

$$
\begin{aligned}
J(X, T) & \ll T^{2+\varepsilon}\left|\sum_{n \geqslant 1} \frac{\lambda_{1 \boxplus \phi}(n)}{n^{1+\varepsilon}} \mathrm{e}\left(3(n X)^{1 / 3}\right) W_{2}\left(\frac{n}{T^{3} / X}\right)\right|+T^{3 / 2+\varepsilon} \\
& \ll \frac{X^{1+\varepsilon}}{T}\left|\sum_{n \geqslant 1} \lambda_{1 \boxplus \phi}(n) \mathrm{e}\left(3(n X)^{1 / 3}\right) W_{3}\left(\frac{n}{T^{3} / X}\right)\right|+T^{3 / 2+\varepsilon},
\end{aligned}
$$

for some inert function $W_{3}$.

We may restrict $T$ such that

$$
Y^{2 / 3-\varepsilon} \ll X^{1 / 3-\varepsilon} \ll T \ll \frac{X^{1+\varepsilon}}{Y}
$$

since otherwise the above sum over $n$ is empty. Combining (3.4), (3.6), (3.7) and (3.8), we arrive at

$$
I(X) \ll \sum_{T \text { dyadic }}\left(\frac{X^{1+\varepsilon}}{T^{2}}\left|\sum_{n \geqslant 1} \lambda_{1 \boxplus \phi}(n) \mathrm{e}\left(3(n X)^{1 / 3}\right) W\left(\frac{n}{T^{3} / X}\right)\right|+T^{1 / 2+\varepsilon}\right) .
$$

Here $X$ on the right hand side of (3.10) should be understood as the original $X u$ in (3.6) with $u \in[1 / 3,3]$. And $W$ is some smooth function such that $\operatorname{supp} W \in[1 / 4,4]$. So we only need to consider the case $n \asymp T^{3} / X$.

Now we make use of the fact that $\lambda_{1 \boxplus \phi}(n)=\sum_{\ell m=n} \lambda_{\phi}(m)$. We can insert a dyadic partition of unit for $\ell$-sum and a dyadic smooth partition of unit for $m$-sum. Hence it suffices to estimate the following sum

$$
B(L, M)=\sum_{\substack{\ell \sim L \\ L M \asymp \frac{T^{3}}{X}}} \sum_{\substack{m \asymp M \\ \text { d }}} \lambda_{\phi}(m) \mathrm{e}\left(3(\ell m X)^{1 / 3}\right) V\left(\frac{m}{M}\right) .
$$

Now we consider two cases.

Case 1. $L \gg T^{359 / 228} X^{-97 / 152}$. We then rewrite $B(L, M)$ as

$$
B(L, M)=\sum_{m \asymp M} \lambda_{\phi}(m) V\left(\frac{m}{M}\right)\left(\sum_{\substack{\ell \sim L \\ L M \asymp \frac{T^{3}}{X}}} \mathrm{e}\left(3(\ell m X)^{1 / 3}\right)\right) .
$$

For the inner sum over $\ell$ we apply the method of exponent pairs with A-process (see for example [6, Chapter 3]), by taking the exponent pair $(p, q)$ as

$$
(p, q)=\left(\frac{k}{2 k+2}, \frac{k+h+1}{2 k+2}\right)=\left(\frac{13}{194}+\varepsilon, \frac{76}{97}+\varepsilon\right)
$$


where $(k, h)=\left(\frac{13}{84}+\varepsilon, \frac{55}{84}+\varepsilon\right)$ is also an exponent pair due to Bourgain [3, Theorem 6]. Hence we obtain

$$
\begin{aligned}
B(L, M) & \ll \sum_{m \asymp M}\left|\lambda_{\phi}(m)\right|\left|\sum_{\substack{\ell \sim L \\
L M \asymp \frac{T^{3}}{X}}} \mathrm{e}\left(3(\ell m X)^{1 / 3}\right)\right| \\
& \ll T^{\varepsilon} M \cdot(T / L)^{p} L^{q} \\
& \ll T^{1195 / 456+\varepsilon} X^{-249 / 304} .
\end{aligned}
$$

Case 2. $L \ll T^{359 / 228} X^{-97 / 152}$. We rewrite $B(L, M)$ as

$$
B(L, M)=\sum_{\ell \sim L}\left(\sum_{\substack{m \asymp M \\ L M \asymp \frac{T^{3}}{X}}} \lambda_{\phi}(m) \mathrm{e}\left(3(\ell m X)^{1 / 3}\right) V\left(\frac{m}{M}\right)\right) .
$$

For the inner sum over $m$, we employ a result of Jutila (see [9, Theorem 4.6]). It is easy to verify that $M^{3 / 4} \ll T \ll M^{3 / 2}$ for $L \ll T^{359 / 228} X^{-97 / 152}$ and

$$
X^{165 / 346} \leqslant T \leqslant X^{3 / 5} \text {. }
$$

Therefore

$$
\begin{aligned}
B(L, M) & \ll \sum_{\ell \sim L}\left|\sum_{\substack{m \asymp M \\
L M \asymp \frac{T^{3}}{X}}} \lambda_{\phi}(m) \mathrm{e}\left(3(\ell m X)^{1 / 3}\right) V\left(\frac{m}{M}\right)\right| \\
& \ll L \cdot T^{1 / 3} M^{1 / 2} \\
& \ll T^{1195 / 456+\varepsilon} X^{-249 / 304} .
\end{aligned}
$$

Combining (3.10), (3.11) and (3.13), we have the following upper bound when $T$ satisfies $X^{165 / 346} \leqslant T \leqslant X^{1 / 2+\delta+\varepsilon}$ :

$$
I(X) \ll \frac{X^{1+\varepsilon}}{T^{2}} \cdot T^{1195 / 456+\varepsilon} X^{-249 / 304}+T^{1 / 2+\varepsilon} \ll X^{1 / 2-\delta+\varepsilon},
$$

with $\delta \leqslant 4 / 739 \approx 0.00541 \ldots$. If $X^{1 / 3-\varepsilon} \leqslant T \leqslant X^{165 / 346}$, we use the trivial bound to get

$$
I(X) \ll \frac{X^{1+\varepsilon}}{T^{2}} \cdot \frac{T^{3}}{X}+T^{1 / 2+\varepsilon} \ll T X^{\varepsilon} \ll X^{165 / 346+\varepsilon} \ll X^{1 / 2-\delta+\varepsilon} .
$$

Finally, putting together the above estimates (3.3), (3.9), (3.14) and (3.15), one can easily complete the proof of Theorem 1.

Remark 3. The exponent pair $\left(\frac{13}{194}+\varepsilon, \frac{76}{97}+\varepsilon\right)$ is the best known exponent pair we find for our problem. We essentially need to choose an exponent pair $(p, q)$ to minimize $\frac{5+5 p-2 q}{11+8 p-5 q}$.

\section{REFERENCES}

[1] F. V. Atkinson. A divisor problem. Quart. J. Math. (Oxford) 12(1941), 193-200.

[2] V. Blomer, R. Khan, and M. Young. Distribution of mass of holomorphic cusp forms. Duke Math. J. 162 (2013), no. 14, 2609-2644.

[3] J. Bourgain. Decoupling exponential sums and the Riemann zeta function, J. Amer. Math. Soc. 30 (2017), no. 1, 205-224.

[4] J.B. Friedlander and H. Iwaniec, Incomplete Kloosterman sums and a divisor problem, Ann. of Math. (2) 121 (1985), no. 2, 319-350. (with an appendix by B. J. Birch and E. Bombieri). 
[5] J.B. Friedlander and H. Iwaniec. Summation formulae for coefficients of $L$-functions. Canad. J. Math. 57 (2005), no. 3, 494-505.

[6] S.W. Graham and G. Kolesnik. Van der Corput's method of exponential sums. London mathematical society lecture note series, Vol. 126. Cambridge university press, 1991.

[7] B. Huang. On the Rankin-Selberg problem. ArXiv preprint (2020), arXiv:2002.00591.

[8] H. Iwaniec and E. Kowalski. Analytic number theory, volume 53 of American Mathematical Society Colloquium Publications. American Mathematical Society, Providence, RI, 2004.

[9] M. Jutila. Lectures on a method in the theory of exponential sums. Tata Institute of Fundamental Research Lectures on Mathematics and Physics, vol. 80, Published for the Tata Institute of Fundamental Research, Bombay; by Springer-Verlag, Berlin, 1987.

[10] E. Kowalski, Ph. Michel, and W. Sawin. Bilinear forms with Kloosterman sums and applications. Ann. of Math. (2) 186 (2017), No. 2, pp. 413-500.

[11] Y. Lin and Q. Sun. Analytic twists of $\mathrm{GL}_{3} \times \mathrm{GL}_{2}$ automorphic forms. ArXiv preprint (2019), arXiv:1912.09772.

BingRONG HuANG

Data Science Institute and School of Mathematics, Shandong University, Jinan, 250100

Shandong, P. R. China

E-mail address: brhuang@sdu.edu.cn

YONGXIAO LIN

EPFL SB MATHGeOM TAN, Station 8, CH-1015, Lausanne, Switzerland

E-mail address: yongxiao.lin@epfl.ch

ZHIWEI WANG

School of Mathematics, Shandong University, Jinan, 250100 Shandong, P. R. China

E-mail address: zhiwei.wang@sdu.edu.cn 